$10 \% 0^{6^{6^{2}}}(1)$

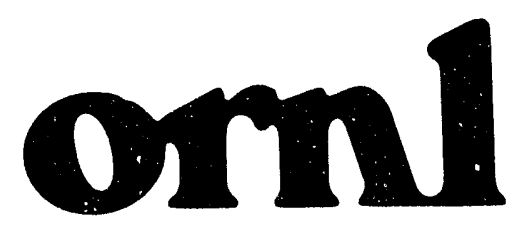

OAK RIDGE

NATIONAL

LABORATORY

MARTH MARUETRA
Reducing Communication Costs in the Conjugate Gradient Algorithm on Distributed Memory Multiprocessors

E. F. D'Azevedo

C. H. Romine 
This report has been reproduced directly from the best available copy.

Available to DOE and DOE contractors from the Office of Scientific and Technical Information, P.O. Box 62, Oak Ridge, TN 37831 ; prices available from (615) 576-8401, FTS 626-8401.

Available to the public from the National Technical Information Service, U.S. Department of Commerce, 5285 Port Royal Rd., Springfield, VA 22161.

This report was prepared as an account of work sponsored by an agency of the United States Government. Neither the United States Government nor any agency thereof, nor any of their employees, makes any warranty, express or implied, or assumes any legal liability or responsibility for the accuracy, completeness, or usefulness of any information, apparatus, product, or process disclosed, or represents that its use would not infringe privately owned rights. Reference herein to any specific commercial product, process, or service by trade name, trademark, manufacturer, or otherwise, does not necessarily constitute or imply its endorsement, recommendation, or favoring by the United States Government or any agency thereof. The views and opinions of authors expressed herein do not necessarily state or reflect those of the United States Government or any agency thereot. 
ORNL/TM-12192

Engineering Physics and Mathematics Division

Mathematical Sciences Section

ORNL/TM- 12192

DE93 000562

\title{
REDUCING COMMUNICATION COSTS IN THE \\ CONJUGATE GRADIENT ALGORITHM ON \\ DISTRIBUTED MEMORY MULTIPROCESSORS
}

\author{
E. F. D'Azevedo \\ C. H. Romine \\ Mathematical Sciences Section \\ Oak Ridge National Laboratory \\ P.O. Box 2008, Bldg. 6012 \\ Oak Ridge, TN 37831-6367
}

Date Published: September 1992

Research supported by the Applied Mathematical Sciences subprogram of the Office of Energy Research, U.S.

Department of Energy

Prepared by the

Oak Ridge National Laboratory

Oak Ridge, Tennessee 37831

managed by

Martin Marietta Energy Systems, Inc.

for the

U.S. DEPARTMENT OF ENERGY

under Contract No. DE-AC05-84OR21400 


\section{Contents}

1 Introduction . . . . . . . . . . . . . . . . . 1

2 The conjugate gradient algorithm ................ 2

3 The Lanczos algorithm . . . . . . . . . . . . . . . . . . . . 4

4 Numerical experiments on stability ............... . . 7

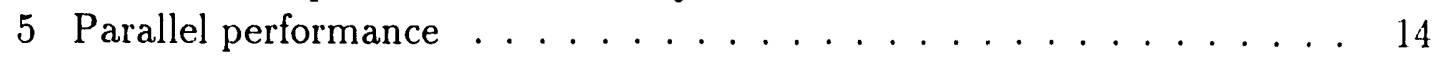

6 Conclusion . . . . . . . . . . . . . . . . . . . 16

7 References ........................... 16 


\section{List of Figures}

4.1 Classical CG on Test 1 . . . . . . . . . . . . . . . 9 9

4.2 Modified CG on Test 1 . . . . . . . . . . . . . 9

4.3 Eijkhout Rearrangement on Test 1 . . . . . . . . . . . . 10

4.4 Meurant Rearrangement on Test 1 . . . . . . . . . . . . 10

4.5 Lanczos Rearrangement on Test 1 . . . . . . . . . . . 11

4.6 Classical CG on Tests 2-4 ................. 11

4.7 Modified CG on Tests 2-4 . . . . . . . . . . . . . . 12

4.8 Eijkhout Rearrangement on Tests 2-4 . . . . . . . . . . . 12

4.9 Meurant Rearrangement on Tests 2-4 . . . . . . . . . . . . . 13

4.10 Lanczos Rearrangement on Tests 2-4 . . . . . . . . . . . . 13 


\title{
REDUCING COMMUNICATION COSTS IN THE CONJUGATE GRADIENT ALGORITHM ON DISTRIBUTED MEMORY MULTIPROCESSORS
}

\author{
E. F. D'Azevedo \\ C. H. Romine
}

\begin{abstract}
The standard formulation of the conjugate gradient algorithm involves two inner product computations. The results of these two inner products are needed to update the search direction and the computed solution. In a distributed memory parallel environment, the computation and subsequent distribution of these two values requires two separate communication and synchronization phases. In this paper, we present a mathematically equivalent rearrangement of the standard algorithm that reduces the number of communication phases. We give a second derivation of the modified conjugate gradient algorithm in terms of the natural relationship with the underlying Lanczos process. We also present empirical evidence of the stability of this modified algorithm.
\end{abstract}




\section{Introduction}

The conjugate gradient ( $\mathrm{CG}$ ) method is an effective iterative method for solving large sparse symmetric positive definite systems of linear equations. It is robust and, coupled with an effective preconditioner [19], is gencrally able to achieve rapid convergence to an accurate solution.

One drawback of the standard formulation of the conjugate gradient algorithm on distributed memory parallel machines is that it involves the computation of two separate inner products of distributed vectors. Moreover, the first inner product must be completed before the data are arailable for computing the second inner product. Hence, a distributed menory implementation of the standard conjugate gradient method has two separate communication phases for these two inner products. Since communication is quite expensive on the current generation of distributed memory multiprocessors, it is desirable to reduce the communication overhead by combining these two communication phases into one.

Saad $[16,17]$ has shown one rearrangement of the computation that eliminates a communication phase by computing $\left\|r_{k}\right\|_{2}$ based on the relationship

$$
\left\|r_{k+1}\right\|_{2}^{2}=\alpha_{k}^{2}\left\|A p_{k}\right\|_{2}^{2}-\left\|r_{k}\right\|_{2}^{2}
$$

to be numerically unstable. Meurant [11] proposed using (1.1) as a predictor for $\left\|r_{k+1}\right\|$ and reevaluate the actual norm on the next iteration with an extra inner product. Van Rosendale [20] has proposed without numerical results an $m$-step) conjugate gradient algorithm to increase parallelism.

The conjugate gradient algorithm is known to be closely related to the Lanczos algorithm for tridiagonalizing a matrix [5]. Paige [12,13,14] has done detailed analysis to show some variants of the Lanczos algorithm are unstable. Strakos [18] and Greenbaum [6,7] have considered the close connection between the Lanczos and CG algorithm in the analysis of stability of CG computations under perturbations in finite arithmetic.

In $\S 2$, we present a rearrangement of the conjugate gradient computation that 
eliminates one communication phase by computing both inner products at once. We show a natural association between this rearrangement and the Lanczos algorithm in $\S 3$. A discussion of how this rearrangement of the computation affects the stability properties of the conjugate gradient algorithm and some MATLAB numerical experiments on the effectiveness of the rearrangement are included in $\S 4$.

\section{The conjugate gradient algorithm}

We begin by reviewing the standard conjugate gradient procedure $[2,9]$ for solving the linear system

$$
A x=b .
$$

For simplicity, we assume a zero initial guess, and residual vector $r_{1}=b$, with $\langle x, y\rangle=x^{t} y$ as the usual inner product.

For $k=1,2, \ldots$

$$
\begin{aligned}
\gamma_{k} & =\left\langle r_{k}, r_{k}\right\rangle \\
\beta_{k} & =\gamma_{k} / \gamma_{k-1} \quad\left(\beta_{1}=0\right) \\
p_{k} & =r_{k}+\beta_{k} p_{k-1} \quad\left(p_{1}=r_{1}\right) \\
v_{k} & =A p_{k} \\
\sigma_{k} & =\left\langle p_{k}, v_{k}\right\rangle \\
\alpha_{k} & =\gamma_{k} / \sigma_{k} \\
x_{k+1} & =x_{k}+\alpha_{k} p_{k} \\
r_{k+1} & =r_{k}-\alpha_{k} v_{k} .
\end{aligned}
$$

Saad [16,17] and Meurant [11] have considered eliminating the first inner product for $\gamma_{k}=\left\langle r_{k}, r_{k}\right\rangle$. We propose eliminating the second communication phase by finding alternative expressions for $\sigma_{k}$ 


$$
\begin{aligned}
\sigma_{k}= & \left\langle p_{k}, v_{k}\right\rangle=\left\langle p_{k}, A p_{k}\right\rangle \\
= & \left\langle r_{k}+\beta_{k} p_{k-1}, A r_{k}+\beta_{k} v_{k-1}\right\rangle \\
= & \left\langle r_{k}, A r_{k}\right\rangle+\beta_{k}\left\langle r_{k}, v_{k-1}\right\rangle+ \\
& \beta_{k}\left\langle p_{k-1}, A r_{k}\right\rangle+\beta_{k}^{2}\left\langle p_{k-1}, v_{k-1}\right\rangle \\
\sigma_{k}= & \left\langle r_{k}, A r_{k}\right\rangle+2 \beta_{k}\left\langle r_{k}, v_{k-1}\right\rangle+\beta_{k}^{2} \sigma_{k-1} .
\end{aligned}
$$

To further simplify (2.4), we rely on an intrinsic property of the CG procedure, the orthogonality of residual vectors (and equivalently the conjugacy of search directions [9, page 420])

$$
\frac{\left\langle r_{k}, r_{k+1}\right\rangle}{\left\langle r_{k}, r_{k}\right\rangle} \equiv \frac{\left\langle p_{k}, A p_{k+1}\right\rangle}{\left\langle p_{k}, A p_{k}\right\rangle}=0
$$

(Eijkhout [4] suggests performing an extra inner product to explicitly evaluate $\left\langle r_{k}, v_{k-1}\right\rangle$ for improved stability prop rties.) Thus from (2.3)

$$
\begin{aligned}
r_{k} & =r_{k-1}-\alpha_{k-1} v_{k-1} \\
\left\langle r_{k}, r_{k}\right\rangle & =\left\langle r_{k}, r_{k-1}\right\rangle-\alpha_{k-1}\left\langle r_{k}, v_{k-1}\right\rangle \\
\gamma_{k} & =0-\alpha_{k-1}\left\langle r_{k}, v_{k-1}\right\rangle .
\end{aligned}
$$

Therefore by (2.4), (2.6) and $\beta_{k}=\gamma_{k} / \gamma_{k-1}$,

$$
\begin{aligned}
& \sigma_{k}=\left\langle r_{k}, A r_{k}\right\rangle+2 \beta_{k}\left(-\gamma_{k} / \alpha_{k-1}\right)+\beta_{k}^{2} \sigma_{k-1} \\
& \sigma_{k}=\delta_{k}-\beta_{k}^{2} \sigma_{k-1}, \text { where } \delta_{k}=\left\langle r_{k}, A r_{k}\right\rangle .
\end{aligned}
$$

We propose the following rearrangement of the conjugate gradient procedure. First initialize $\sigma_{1}$ and $v_{1}$, by performing one step of the standard algorithm

$$
\begin{aligned}
& r_{1}=b, \quad \gamma_{1}=\left\langle r_{1}, r_{1}\right\rangle, \quad p_{1}=r_{1}, \quad v_{1}=A p_{1} \\
& \sigma_{1}=\left\langle p_{1}, v_{1}\right\rangle, \quad x_{2}=\left(\gamma_{1} / \sigma_{1}\right) p_{1}
\end{aligned}
$$


For $k=2,3, \ldots$

$$
\begin{aligned}
s_{k} & =A r_{k} \\
\text { Compute } \gamma_{k} & =\left\langle r_{k}, r_{k}\right\rangle \text { and } \delta_{k}=\left\langle r_{k}, s_{k}\right\rangle \\
\beta_{k} & =\gamma_{k} / \gamma_{k-1} \\
p_{k} & =r_{k}+\beta_{k} p_{k-1} \\
v_{k} & =s_{k}+\beta_{k} v_{k-1} \quad\left(v_{k} \equiv A p_{k}\right) \\
\sigma_{k} & =\delta_{k}-\beta_{k}^{2} \sigma_{k-1} \\
\alpha_{k} & =\gamma_{k} / \sigma_{k} \\
x_{k+1} & =x_{k}+\alpha_{k} p_{k} . \\
r_{k+1} & =r_{k}-\alpha_{k} v_{k} .
\end{aligned}
$$

Note that the above procedure requires extra storage for the vector $s_{k}$ and extra work in updating the vector $v_{k}$; in contrast to Meurant's rearrangement, only two inner products and one matrix vector multiply are performed.

\section{The Lanczos algorithm}

In this section we present a natural derivation of the modified CG rearrangement from the Lanczos process. The Lanczos process is a tridiagonalization procedure to find orthogonal $Q$ and tridiagonal $T$ such that

$$
Q^{t} A Q=T=\left[\begin{array}{cccc}
\tilde{\alpha}_{1} & \tilde{\beta}_{1} & & \\
\tilde{\beta}_{1} & \tilde{\alpha}_{2} & \ddots & \\
& \ddots & \ddots & \dot{\beta}_{n-1} \\
& & \tilde{\beta}_{n-1} & \tilde{\alpha}_{n}
\end{array}\right], \quad Q^{t} Q=I
$$

We arrive at the Lanczos algorithm by equating columns in $A Q=Q T$, where $q_{j}$ 's are columns of $Q, Q=\left[q_{1}|\ldots| q_{n}\right]$

$$
A q_{j}=\tilde{\beta}_{j-1} q_{j-1}+\tilde{\alpha}_{j} q_{j}+\tilde{\beta}_{j} q_{j+1}
$$




$$
\begin{aligned}
\tilde{\beta}_{j} q_{j+1} & =u_{j}-\tilde{\alpha}_{j} q_{j}, \quad \text { where } u_{j}=A q_{j}-\dot{\beta}_{j-1} q_{j-1} \\
\left\langle q_{j}, A q_{j}\right\rangle & =\dot{\alpha}_{j}, \quad\left\langle q_{j+1}, A q_{j}\right\rangle=\dot{\beta}_{j} .
\end{aligned}
$$

Paige [12] presents the following stable arrangement of the Lanczos algorithm. Choose $\tilde{r}_{1}$ to be nonzero,

$$
\tilde{\beta}_{1}= \pm\left\|\tilde{r}_{1}\right\|_{2}, \quad q_{1}=\tilde{r}_{1} / \tilde{\beta}_{1}, \quad u_{1}=A q_{\mathrm{i}}
$$

For $j=1,2, \ldots$

$$
\begin{aligned}
\tilde{\alpha}_{j} & =\left\langle q_{j}, A q_{j}\right\rangle=\left\langle\tilde{r}_{j}, A \tilde{r}_{j}\right\rangle /\left\langle\tilde{r}_{j}, \tilde{r}_{j}\right\rangle \\
\tilde{r}_{j+1} & =u_{j}-\tilde{\alpha}_{j} q_{j} \\
\tilde{\beta}_{j+1} & = \pm\left\|\tilde{r}_{j+1}\right\|_{2} \\
q_{j+1} & =\tilde{r}_{j+1} / \tilde{\beta}_{j+1} \\
u_{j+1} & =A q_{j+1}-\tilde{r}_{j} .
\end{aligned}
$$

In the same paper, Paige [12] shows that computing with the equivalent formula $\tilde{\beta}_{j}=\left\langle q_{j+1}, A q_{j}\right\rangle$ for $\left\|\tilde{r}_{j}\right\|_{2}$ leads to poor stability.

It is well known [5, page 370] that the CG algorithm is the Lanczos process where the normalized residual vectors form the $Q$ matrix. From the $C G$ procedure (2.2) we have

$$
p_{1}=r_{1}, \quad p_{j}=r_{j}+\beta_{j} p_{j-1},
$$

or written in matrix form $R_{k}=\left[r_{1}|\ldots| r_{k}\right], P_{k}=\left[p_{1}|\ldots| p_{k}\right]$,

$$
R_{k}=P_{k} L_{k}^{t}, \quad L_{k}=\left[\begin{array}{cccc}
1 & & & \\
-\beta_{2} & 1 & & \\
& \ddots & \ddots & \\
& & -\beta_{k} & 1
\end{array}\right]
$$

Then $Q_{k}=R_{k} \Delta_{k}^{-1}$ is orthogonal, where $\Delta_{k}=\operatorname{diag}\left(\left\|r_{1}\right\|_{2}, \ldots,\left\|r_{k}\right\|_{2}\right)$, and $T_{k}=$ 
$Q_{k}^{t} A Q_{k}$ is tridiagonal,

$$
\begin{aligned}
Q_{k}^{t} A Q_{k}= & \left(R_{k} \Delta_{k}^{-1}\right)^{t} A\left(R_{k} \Delta_{k}^{-1}\right)=\Delta_{k}^{-1} R_{k}^{t} A R_{k} \Delta_{k}^{-1} \\
= & \Delta_{k}^{-1}\left(P_{k} L_{k}^{t}\right)^{t} A\left(P_{k} L_{k}^{t}\right) \Delta_{k}^{-1}=\left(\Delta_{k}^{-1} L_{k}\right)\left(P_{k}^{t} A P_{k}\right)\left(\Delta_{k}^{-1} L_{k}\right)^{t} \\
= & \left(\Delta_{k}^{-1} L_{k}\right) D_{k}\left(\Delta_{k}^{-1} L_{k}\right)^{t}=T_{k} \\
& \left(D_{k}=\operatorname{diag}\left(\sigma_{1}, \ldots, \sigma_{k}\right), \quad \sigma_{j}=\left\langle p_{j}, A p_{j}\right\rangle\right)
\end{aligned}
$$

By equating the entries in (3.6) and (3.1), we have

$$
\tilde{\alpha}_{j}=\frac{\sigma_{j}+\beta_{j}^{2} \sigma_{j-1}}{\gamma_{j}}=\frac{1}{\alpha_{j}}+\frac{\beta_{j}}{\alpha_{j-1}}, \quad \dot{\beta}_{j}=\frac{-\beta_{j+1} \sigma_{j}}{\sqrt{\gamma_{j}} \sqrt{\gamma_{j+1}}}=\frac{-\sqrt{\beta_{j+1}}}{\alpha_{j}}
$$

Note (3.6) with $\tilde{\alpha}_{j}=\left\langle q_{j}, A q_{j}\right\rangle$ gives (2.7)

$$
\begin{aligned}
\tilde{\alpha}_{j} & =\left\langle q_{j}, A q_{j}\right\rangle=\frac{\left\langle r_{j}, A r_{j}\right\rangle}{\left\langle r_{j}, r_{j}\right\rangle}=\frac{\delta_{j}}{\gamma_{j}}=\frac{\sigma_{j}+\beta_{j}^{2} \sigma_{j-1}}{\gamma_{j}} \\
\left\langle r_{j}, A r_{j}\right\rangle & =\delta_{j}=\sigma_{j}+\beta_{j}^{2} \sigma_{j-1} .
\end{aligned}
$$

Golub and Van Loan [5, page 342] present the application of the Lanczos process in solving linear equations, which is equivalent to the CG algorithm

$$
\begin{aligned}
Q_{k}^{t} A Q_{k} y_{k} & =Q_{k}^{t} b, \quad Q_{k} y_{k}=x_{k} \\
T_{k} y_{k} & =\tilde{b}_{k}, \quad \tilde{b}_{k}=Q_{k}^{t} b
\end{aligned}
$$

The solution $x_{k}=Q_{k} y_{k}$ can then be computed from (3.8) by the $L D L^{t}$ factorization (3.6) of $T_{k}$

$$
\begin{aligned}
x_{k} & =Q_{k} T_{k}^{-1} Q_{k}^{t} b=Q_{k} T_{k}^{-1} \dot{b}_{k} \\
& =Q_{k}\left[\left(\Delta_{k}^{-1} L_{k}\right) D_{k}\left(\Delta_{k}^{-1} L_{k}\right)^{t}\right]^{-1} \tilde{b}_{k} \\
& =\left(P_{k} L_{k}^{t} \Delta_{k}^{-1}\right)\left[\Delta_{k} L_{k}^{-t} D_{k}^{-1} L_{k}^{-1} \Delta_{k}\right] \tilde{b}_{k} \\
& =P_{k} a_{k}, \quad \text { where } L_{k} D_{k} a_{k}=\Delta_{k} \tilde{b}_{k}
\end{aligned}
$$


With further simplifications, one can show $a_{k}=\left[\alpha_{1}, \ldots, \alpha_{k}\right]^{t}$ and this process can be rewritten in the more familiar form $x_{k}=x_{k-1}+\alpha_{k} p_{k}$ of the CG algorithm.

We note that the above process requires computation of $\tilde{\beta}_{j}= \pm\left\langle\tilde{r}_{j}, \tilde{r}_{j}\right\rangle$ and $\tilde{\alpha}_{j}=\left\langle\tilde{r}_{j}, A \tilde{r}_{j}\right\rangle /\left\langle\tilde{r}_{j}, \tilde{r}_{j}\right\rangle$; as in the modified CG rearrangement (2.8), both inner products can be computed together. Moreover, the use of the alternative formula $\tilde{\beta}_{j}=\left\langle q_{j+1}, A q_{j}\right\rangle$ for $\left\|\tilde{r}_{j}\right\|_{2}$ leads to instability, as in the case of Saad's rearrangernent (1.1). Hence, algorithm (3.3) with (3.9) is a CG-like algorithm that differs from (2.8) in the computing of $r_{j}$ from $q_{j}$. The formulae

$$
p_{j}=r_{j}+\beta_{j} p_{j-11}, \quad r_{j+1}=r_{j}-\alpha_{j} A p_{j}
$$

from (2.8) can be shown to be equivalent to the three-term recurrence relation (3.2) of the Lanczos algorithm. We have

$$
\begin{aligned}
r_{j+1} & =r_{j}-\alpha_{j} A\left(r_{j}+\beta_{j} p_{j-1}\right)=r_{j}-\alpha_{j} A r_{j}-\left(\alpha_{j} \beta_{j} / \alpha_{j-1}\right)\left(r_{j-1}-r_{j}\right) \\
& =\left(1+\alpha_{j} \beta_{j} / \alpha_{j-1}\right) r_{j}-\alpha_{j} A r_{j}-\left(\beta_{j} \alpha_{j} / \alpha_{j-1}\right) r_{j-1} \\
& =\tilde{\alpha}_{j} \alpha_{j} r_{j}-\alpha_{j} A r_{j}-\left(\beta_{j} \alpha_{j} / \alpha_{j-1}\right) r_{j-1} \\
A r_{j} & =\left(-\beta_{j} / \alpha_{j-1}\right) r_{j-1}+\tilde{\alpha}_{j} r_{j}-\left(1 / \alpha_{j}\right) r_{j+1} .
\end{aligned}
$$

By (3.6) and the relationships $q_{j}=\tilde{r}_{j} / \tilde{\beta}_{j}=r_{j} / \sqrt{\gamma_{j}},(3.10)$ simplifies to (3.2). Thus in this light, the modified CG rearrangement (2.8) is naturally associated with the Lanczos process.

\section{Numerical experiments on stability}

The aim of the following experiments is to determine the stability and convergence properties of the modified conjugate gradient procedures.

We performed a number of MATLAB experiments in solving $A x=b$ by the conjugate gradient procedure to study the convergence behavior on different distributions of eigenvalues of the preconditioned matrix. In Eijkhout's rearrangement, $\left\langle r_{k}, v_{k-1}\right\rangle$ is computed by an extra inner product. Meurant's rearrangement 
is taken from [11] and the Lanczos rearrangement is adapted from [5, page 342] by evaluating the two inner products for $\tilde{\alpha}_{j}$ together as $\tilde{\alpha}_{j}=\left\langle\dot{r}_{j}, A \hat{r}_{j}\right\rangle /\left\langle\tilde{r}_{j}, \dot{r}_{j}\right\rangle$.

\section{Test 1}

The matrices considered have the eigenspectrum used by Strakos [18] and Greenbaum and Strakos [7]

$$
\lambda_{i}=\lambda_{1}+\frac{i-1}{n-1}\left(\lambda_{n}-\lambda_{1}\right) \rho^{n-i}, \quad i=2, \ldots, n, \quad \rho \in(0,1) .
$$

We have used $n=100, \lambda_{1}=1 E-3, \kappa=\lambda_{n} / \lambda_{1}=1 E 5$ and $\rho=0.6,0.8,0.9,1.0$ in the experiments. For $\rho=1$, we have a uniformly distributed spectrum, and $\rho<1$ describes quantitatively the clustering ar $\lambda_{1}$.

\section{Test 2}

The eigenspectrum has a gap, $\{1, \ldots, 50,10051, \ldots, 10100\}$.

\section{Test 3}

The eigenspectrum has double eigenvalues, $\{1,1,2,2, \ldots, 50,50\}$.

\section{Test 4}

The eigenspectrum consists of the roots of the Chebyshev polyromial $T_{n}(x)$ shifted from $[-1,1]$ to the interval $[a, b]$

$$
\lambda_{i}=\frac{(b-a)}{2} \cos \left(\frac{\pi / 2+(i-1) \pi}{n}\right)+\frac{(b+a)}{2}, \quad i=1, \ldots, n .
$$

We have used $n=100, a=1, b=1$ E5.

As done in Hageman and Young [8], Greenbaum [6] and Strakos [18], we operate on diagonal matrices. This procedure is equivalent to representing all vectors over the basis of eigenvectors of matrix $A$. In all cases, a random ${ }^{1}$ right

\footnotetext{
${ }^{1}$ uniform over $[-1,1]$
} 


\section{- 9 -}

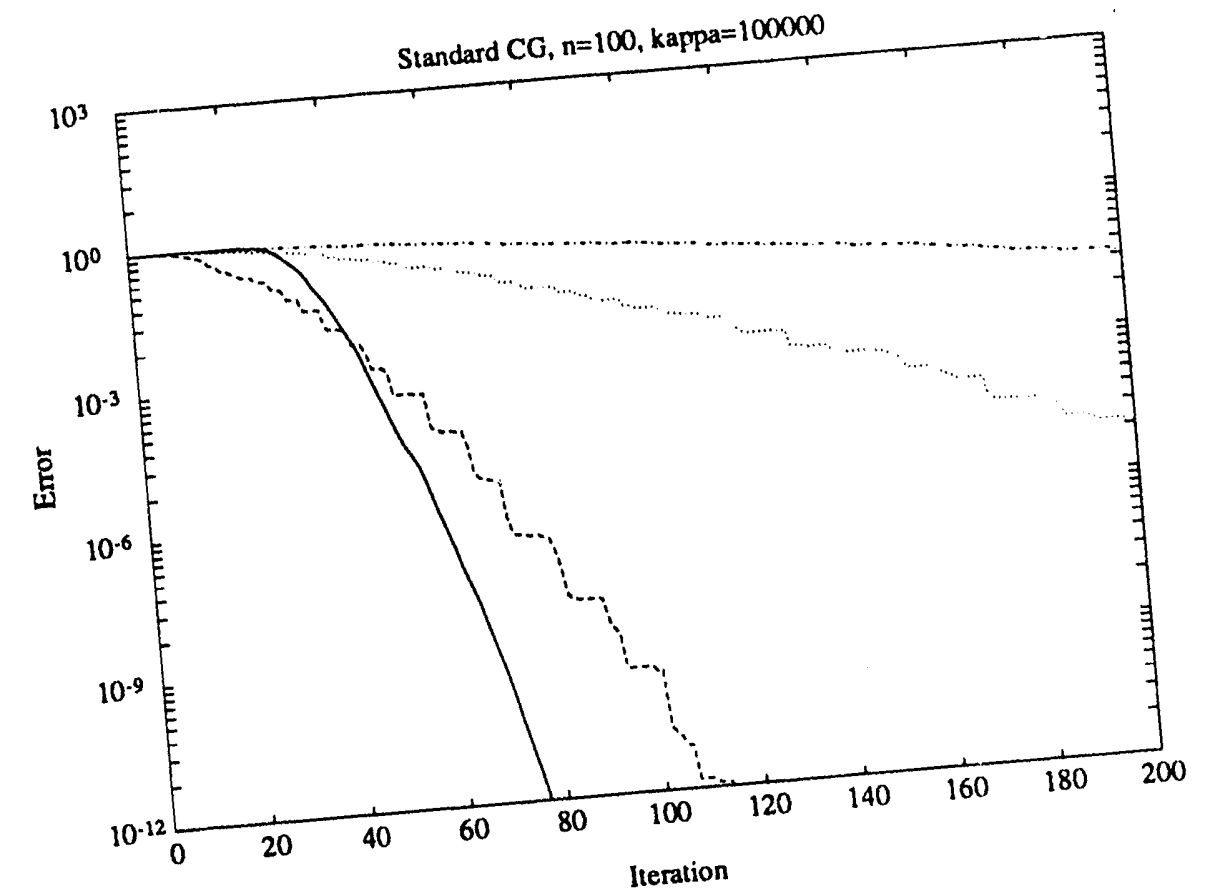

Figure 4.1: Classical CG on Test 1. Dashed curve: $\rho=0.6$; dotted curve: $\rho=0.8$; dash-dot curve: $\rho=0.9$; solid curve: $\rho=1$.

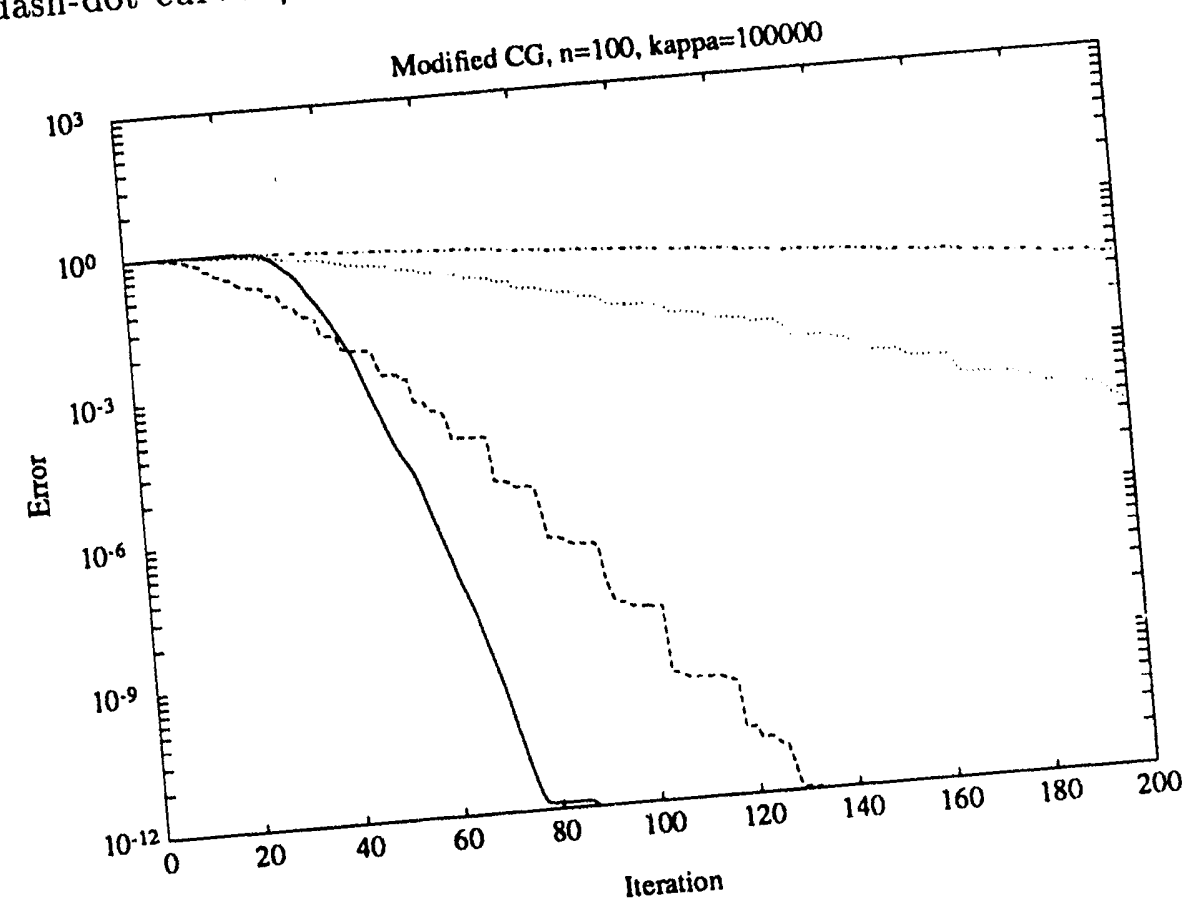

Figure 4.2: Modified CG on Test 1. Dashed curve: $\rho=0.6$; dotted curve: $\rho=0.8$; dash-dot curve: $\rho \doteq 0.9$; solid curve: $\rho=1$. 


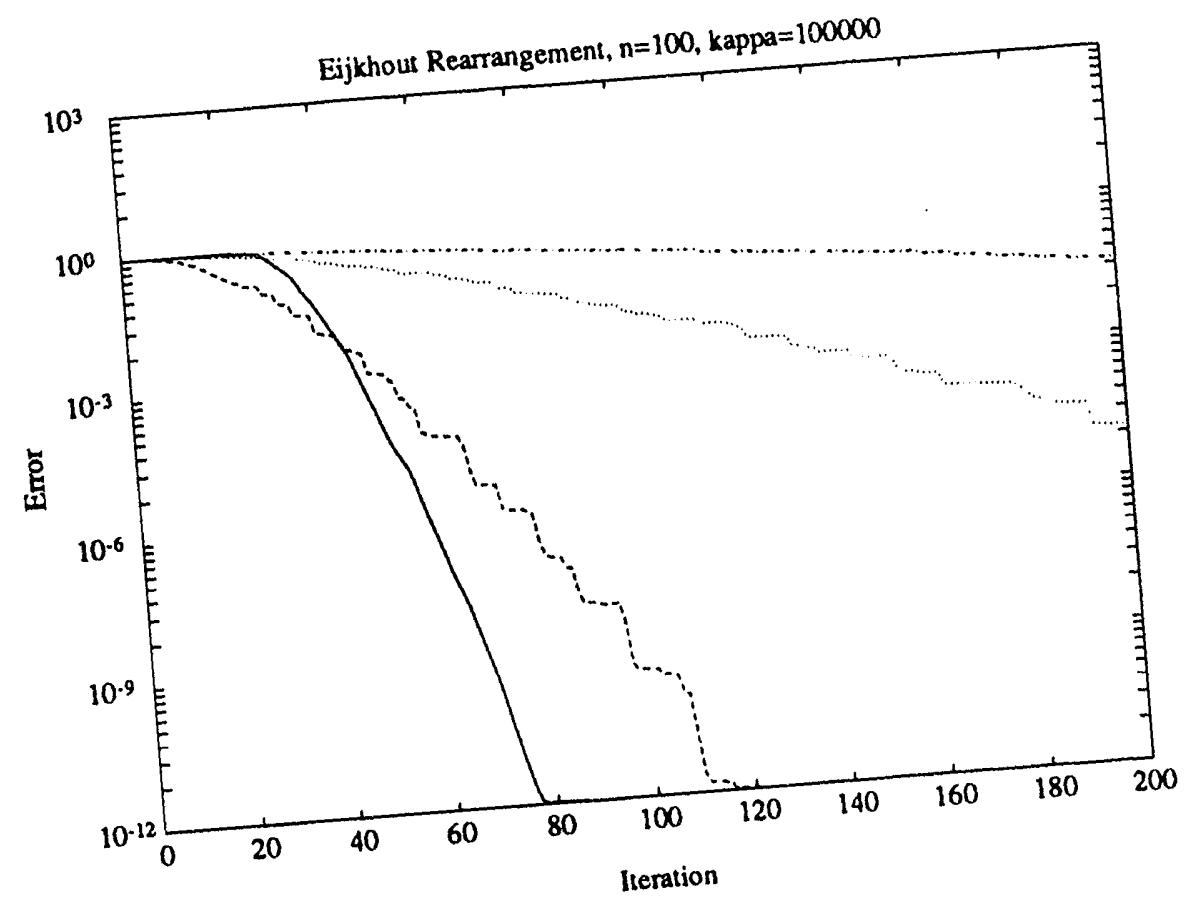

Figure 4.3: Eijkhout Rearrangement on Test 1. Dashed curve: $\rho=0.6$; dotted curve: $\rho=0.8$; dash-dot curve: $\rho=0.9$; solid curve: $\rho=1$.

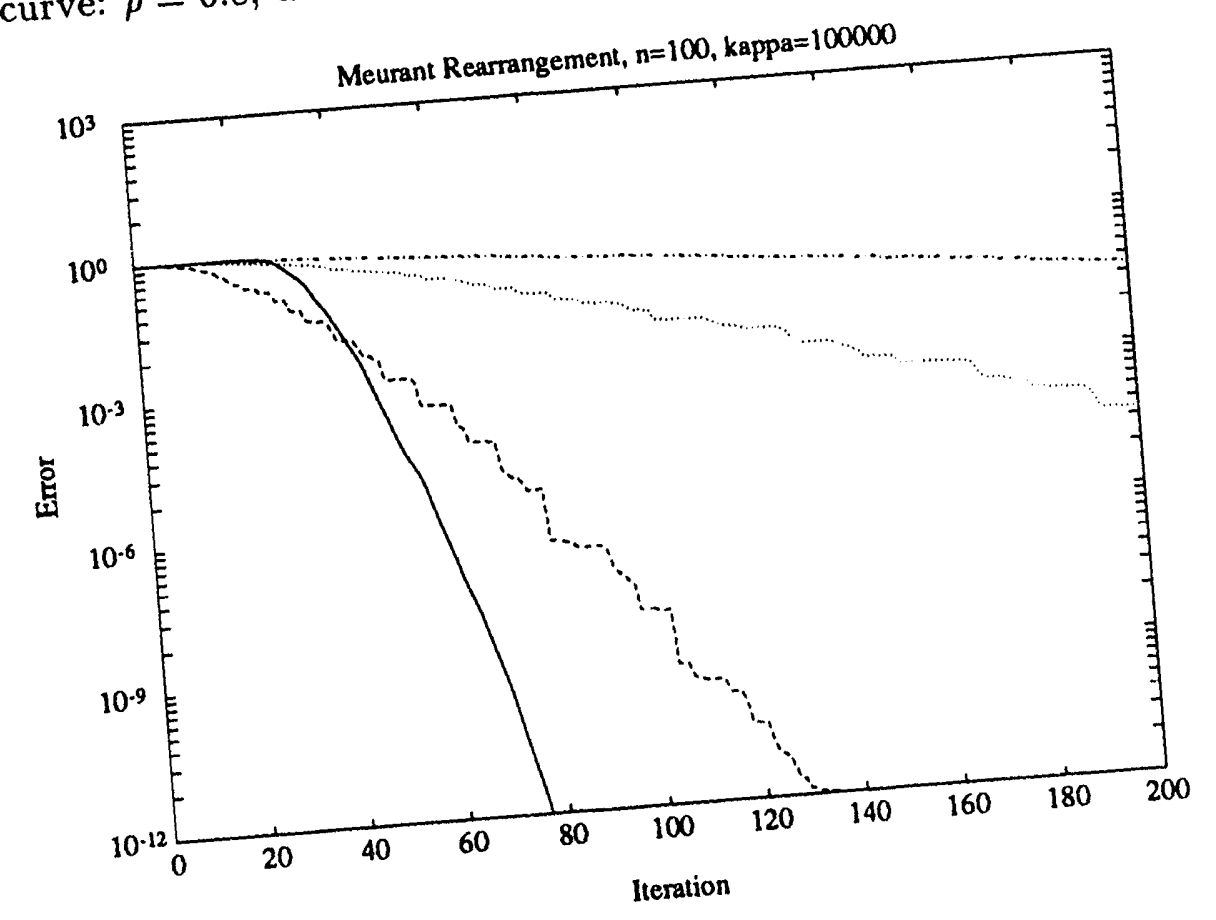

Figure 4.4: Meurant Rearrangement on Test 1. Dashed curve: $\rho=0.6$; dotted curve: $\rho=0.8$; dash-dot curve: $\rho=0.9$; solid curve: $\rho=1$. 


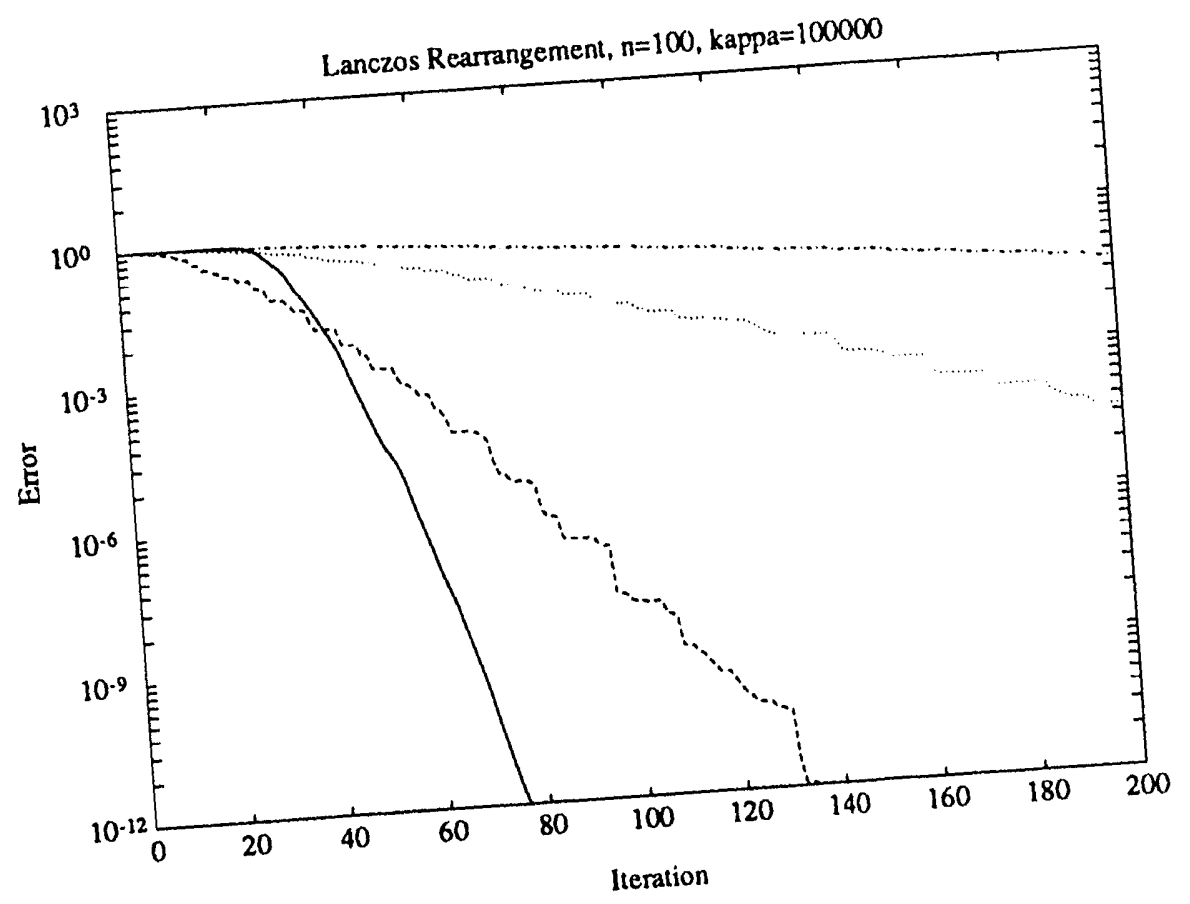

Figure 4.5: Lanczos Rearrangement on Test 1. Dashed curve: $\rho=0.6$; dotted curve: $\rho=0.8$; dash-dot curve: $\rho=0.9$; solid curve: $\rho=1$.

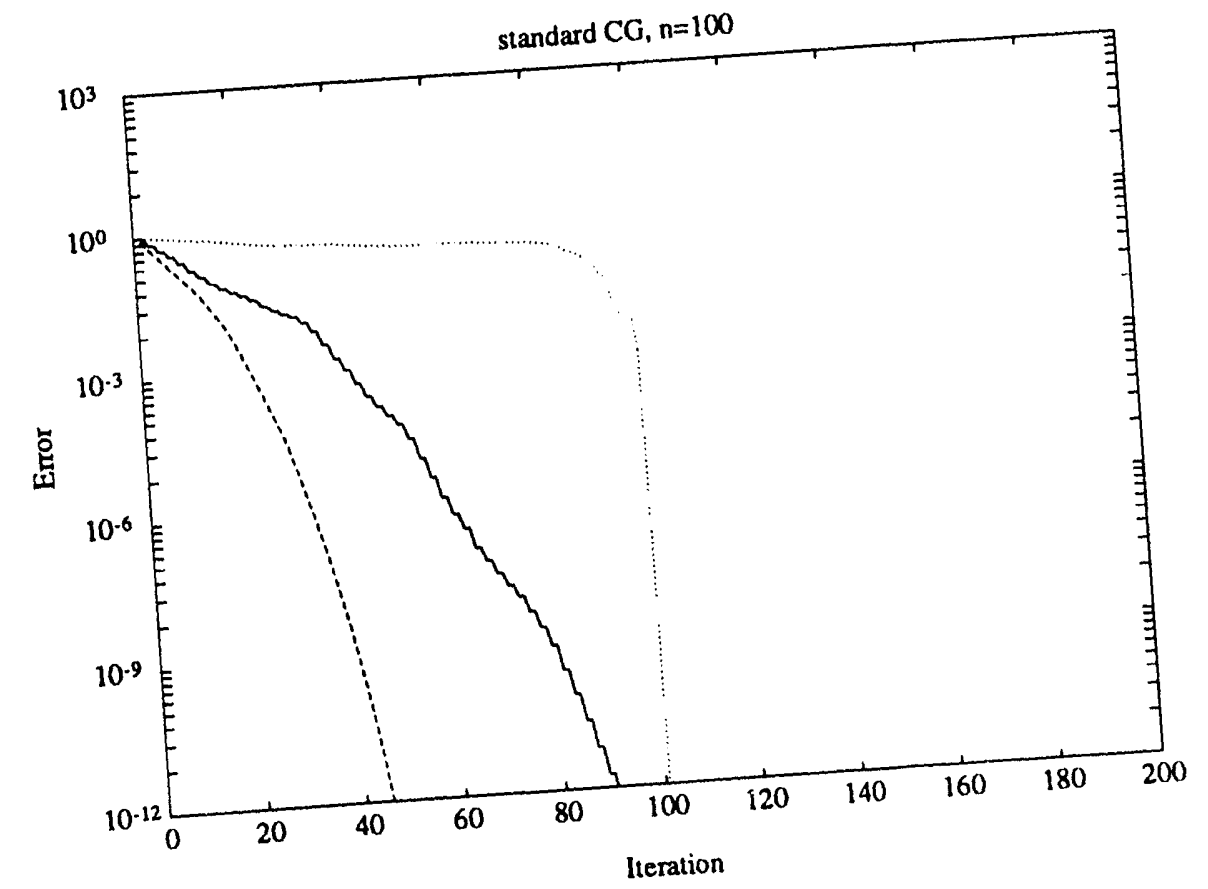

Figure 4.6: Classical CG on Tests 2-4. Solid curve: Test 2; dashed curve: Test 3; dotted curve Test 4. 


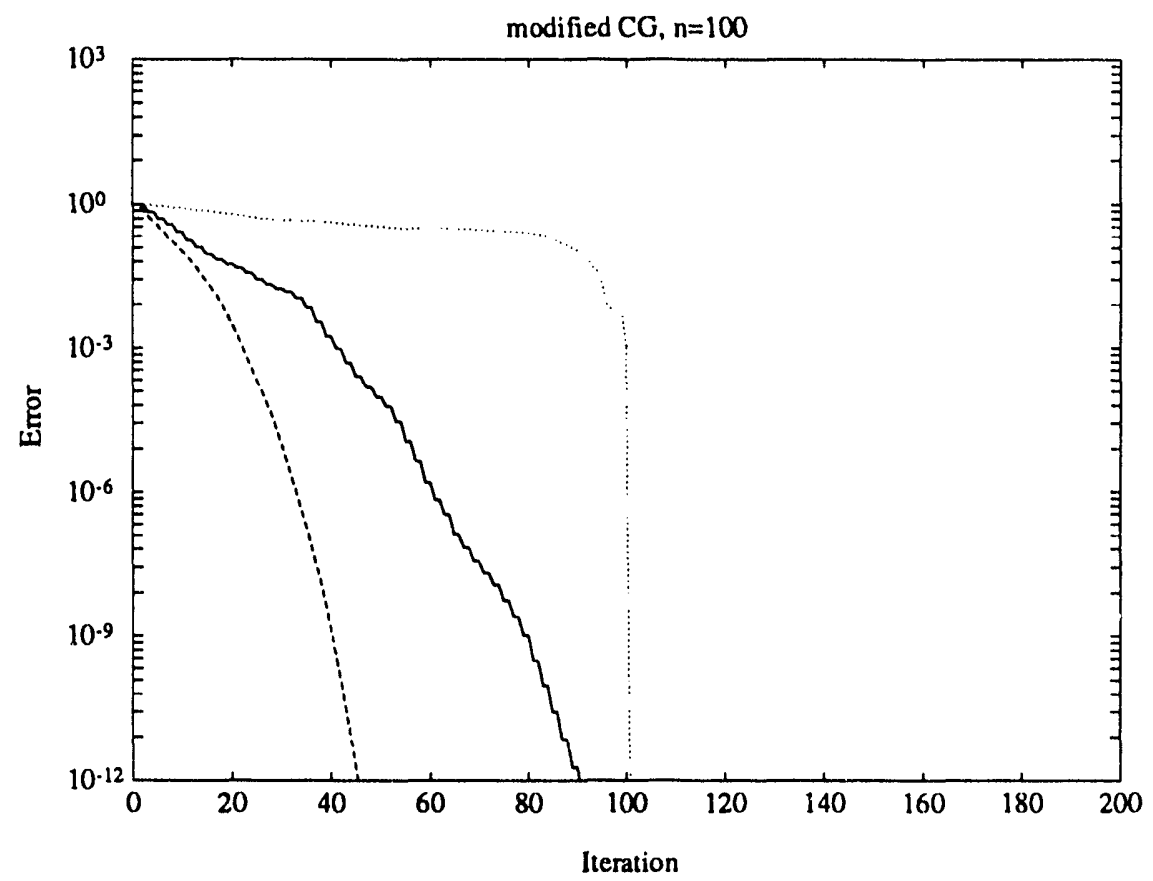

Figure 4.7: $M$ wified CG on Tests 2-4. Solid curve: Test 2; dashed curve: Test 3; dotted curve Test 4.

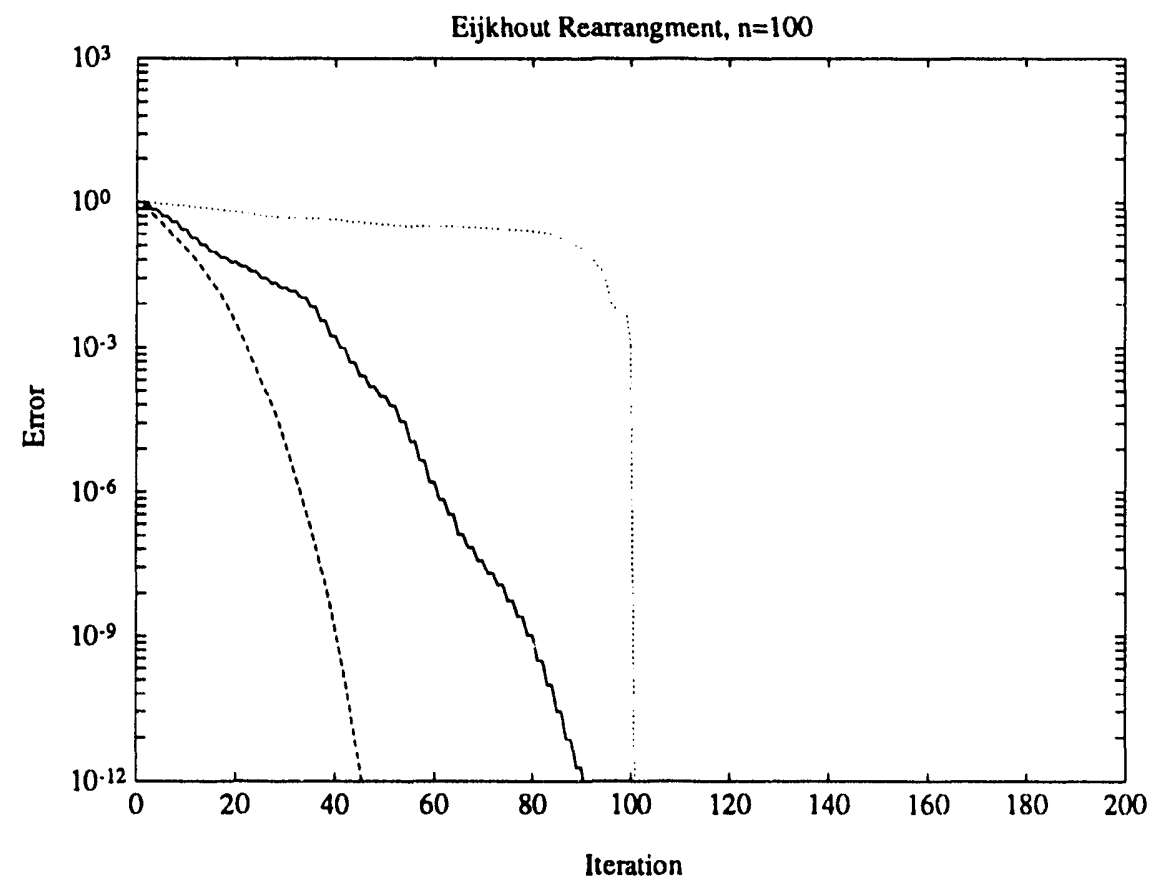

Figure 4.8: Eijkhout Rearrangement on Tests 2-4. Solid curve: Test 2; dashed curve: Test 3 ; dotted curve Test 4 . 


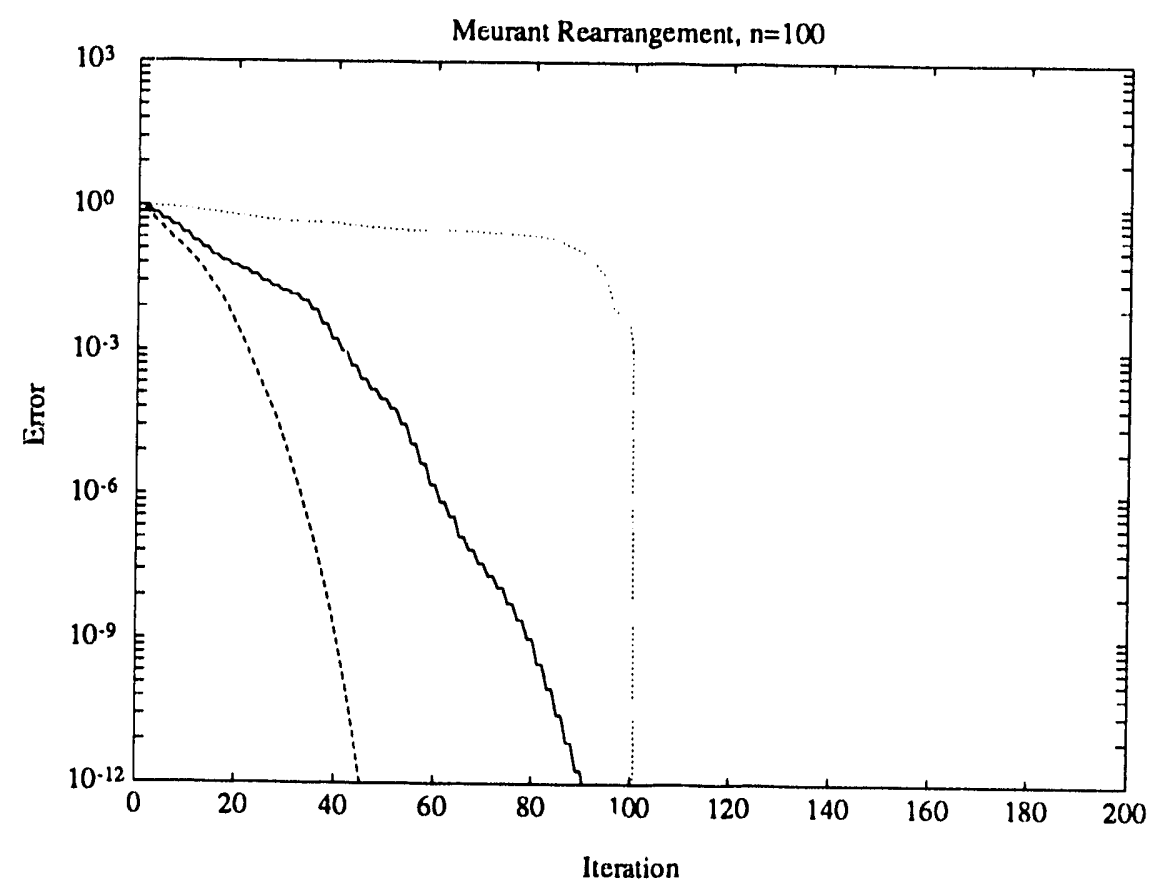

Figure 4.9: Meurant Rearrangement on Tests 2-4. Solid curve: Test 2; dashed curve: Test 3 ; dotted curve Test 4.

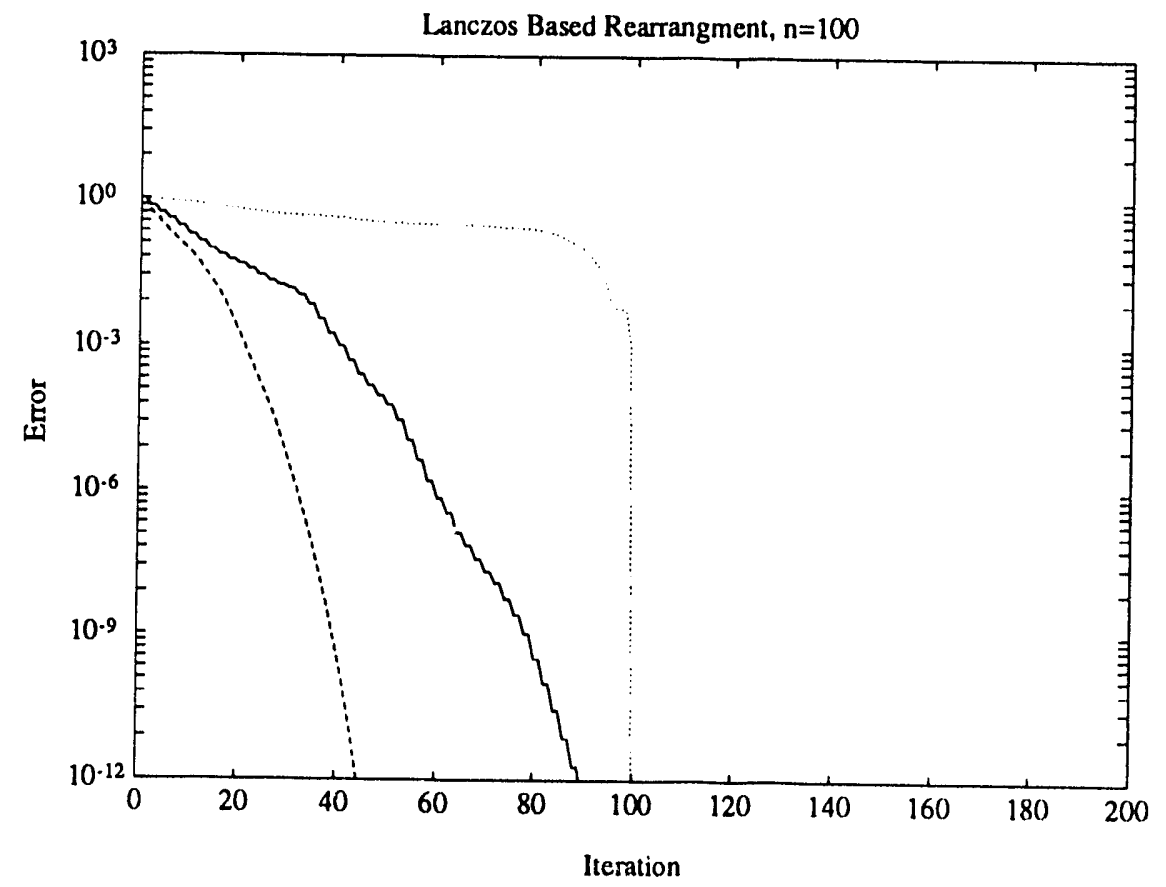

Figure 4.10: Lanczos Rearrangement on Tests 2-4. Solid curve: Test 2; dashed curve: Test 3 ; dotted curve Test 4 . 
hand side and zero initial guess are used.

We display the decrease of $A$-norm of the error at each iteration divided by the $A$-norm of the initial error

$$
\frac{\left\langle\tilde{x}-x_{k}, A\left(\tilde{x}-x_{k}\right)\right\rangle^{1 / 2}}{\left\langle\tilde{x}-x_{0}, A\left(\tilde{x}-x_{0}\right)\right\rangle^{1 / 2}}, \quad \bar{x}=A^{-1} b .
$$

Figures 4.1-4.5, display the convergence results from Test 1. Note that for $\rho=0.8,0.9$ both the standard and modified CG procedures exhibit similar slow convergence behavior. Figures 4.6-4.10 display the convergence results on Tests 2-4. For Test 1 with $\rho=0.6$, the standard CG algorithm shows the best convergence properties. Eijkhout's rearrangement has slightly better stability properties than modified CG. The other results are essentially the same.

All the results on Tests 2-4 again show similar convergence behavior among the standard CG and the different rearrangements of $C G$.

\section{Parallel performance}

To gauge the effectiveness of the modified $\mathrm{CG}^{\prime}$ procedure, we performed a number of experiments in comparing the run-time in standard CG and modified CG. The test matrices are chosen from the Harwell-Boeing Test Collection [3]. The experiments are performed on 16 nodes of the iPSC/860 hypercube. Each matrix is first reordered by the bandwidth reducing Reverse Cuthill-McKee ordering [10]. The matrix is then equally block partitioned by rows and distributed across the processors in ELLPACK format [15]. In all cases, a random right hand side and zero initial guess are used, and convergence is assumed when

$$
\left\|r_{k}\right\|_{2} \leq 10^{-8}\left\|r_{0}\right\|_{2}
$$

The conjugate gradient procedure is rarely used without some form of preconditioning to accelerate convergence. In the tests described below, we use a block preconditioner derived as follows: Let $A_{i}$ be the diagonal block of the 
Table 5.1: Description of test problems.

\begin{tabular}{r|r|r|l}
\hline Problem & Order & Nonzeros & Description \\
\hline BCSSTK13 & 2003 & 11973 & Fluid Flow Generalized Eigenvalues \\
BCSSTK14 & 1806 & 32630 & Root of Omni Coliseum, Atlanta \\
BCSSTK15 & 3948 & 60882 & Module of an Offshore Platform \\
BCSSTK18 & 11948 & 80519 & R.E. Ginna Nuclear Power Station \\
\hline
\end{tabular}

Table 5.2: Timing results.

\begin{tabular}{r|r|r||r|r}
\hline & \multicolumn{2}{|c||}{ standard CG } & \multicolumn{2}{c}{ modified CG } \\
\hline Problem & Iterations & Time & Iterations & Time \\
\hline BCSSTK13 & 1007 & 19.56 & 1007 & 16.99 \\
BCSSTK14 & 232 & 2.72 & 232 & 2.35 \\
BCSSTK15 & 376 & 7.60 & 376 & 6.72 \\
BCSSTK18 & 697 & 34.55 & 697 & 32.80 \\
\hline
\end{tabular}

matrix $A$ contained in processor $i$, and write $A_{i}=L_{i}+D_{i}+L_{i}^{t}$ where $L_{i}$ is strictly lower triangular and $D_{i}$ is diagonal. Then the preconditioning matrix $M$ is $M=\operatorname{diag}\left(M_{1}, M_{2}, \ldots, M_{p}\right)$, where $M_{i}=\left(L_{i}+D_{i}\right) D_{i}^{-1}\left(L_{i}+D_{i}\right)^{t}$. As shown in Axelsson and Barker [1], this corresponds to each processor doing a single SSOR step (with $\omega=1$ ) on its diagonal block $A_{i}$. This preconditioner requires no added communication among the processors when implemented in parallel

Table 5.1 is a brief description of the problems selected from the HarwellBoeing Test Collection. Table 5.2 shows the number of iterations and time (in seconds) required to solve the corresponding problems. In all cases, the modified $\mathrm{CG}$ shows an improvement in the time required for solution, ranging from $5 \%$ to $13 \%$. Moreover, the modified CG rearrangement shows no unstable behavior since it takes almost exactly the same number of iterations as standard C.G. 


\section{Conclusion}

We have presented a rearrangement of the standard conjugate gradient procedure that eliminates one synchronization point by performing two inner products at once. The rearrangement has a natural connection with the Lanczos process for solving linear equations. Although not a proof, MATLAB simulations indicate that the rearrangement is stable. Moreover, computational experiments using parallel versions of both the modified and standard conjugate gradient algorithms show that the modified version reduces the execution time by as much as $13 \%$ on an Intel iPSC/860 with 16 processors.

\section{References}

[1] O. Axelsson and V. A. Barker, Finite Element Solution of Boundary Value Problems, Academic Press, 1984.

[2] P. Concus, G. Golub, And D. O'Leary, A generalized conjugate gradient method for the numerical solution of elliptic partial differential equations, in Sparse Matrix Computations, J. Bunch and D. Rose, eds., Academic Press, New York, 1976, pp. 309-322.

[3] I. S. Duff, R. G. Grimes, and J. G. Lewis, Sparse matrix test problems, ACM Transactions on Mathematical Software, 15 (89), pp. 1-14.

[4] V. EiskhoUT. personal communication.

[5] G. H. Golub and C. F. van Loan, Matrix Computations, John Hopkins University Press, Baltimore, Maryland, 1983.

[6] A. Greenbaum, Behavior of slightly perturbed Lanczos and conjugategradient recurrences, J. Linear Algebra Appl., 113 (1989), pp. 7-63.

[7] A. Greenbaum and Z. Strakos, Predicting the behavior of finite precision Lanczos and conjugate gradient computations, SIAM J. Matrix Anal. Appl., 13 (1992), pp. 121-137. 
[8] L. A. Hageman and D. M. Young, Applied Iterative Methods, Academic, New York, 1981.

[9] M. R. Hestenes AND E. Stiefel, Methods of conjugate gradients for solving linear systems, J. Res. Nat. Bur. Standards, 49 (1952).

[10] J. W.-H. LiU AND A. H. Sherman, Comparative analysis of the CuthillMcliee and the reverse Cuthill-McKee ordering algorithms for sparse matrices, SIAM Journal on Numerical Analysis, 13 (1975), pp. 198-213.

[11] G. MEurant, Multitasking the conjugate gradient method on the CRAY $X-M P / 48$, Parallel Comput., 5 (1987), pp. 267-280.

[12] C. C. PAIGE, Computational variants of the Lanczos method for eigenproblem, J. Inst. Maths. Applics., 10 (1972), pp. 373-381.

[13] _- Error analysis of the Lanczos algorithm for tridiagonalizing a symmetric matrix, J. Inst. Maths. App'ics., 18 (1976), pp. 341-.349.

[14] _- Accuracy and effectiveness of the Lanczos algorithm for the symmetric eigenproblem, J. Linear Algebra Appl., 34 (1980), pp. 235-258.

[15] J. R. Rice ANd R. F. Boisvert, Solving Elliptic Problems Using ELLPACK, Springer-Verlag, New York, 1985.

[16] Y. SAAD, practical use of polynomials preconditionings for the conjugate gradienl method, SIAM J. Sci. Statist. Comput., 6 (1985), pp. 865-881.

[17] Y. SAAD, Krylov subspace methods on supercontputers, SIAM J. Sci. Statist. Comput., 10 (1989), pp. 1200-1232.

[18] Z. STRAKOS, On the real convergence rate of the conjugate gradient method, J. Linear Algebra Appl., 154-156 (1991), pp. 535-549.

[19] H. A. VAN DER Vorst, High performance preconditioning, SIAM J. Sci. Statist. Comput., 10 (1989), pp. 1174-1185. 
[20] J. van Rosendale, Minimizing inner product data dependencies in conjugate gradient iteration, Tech. Report NASA Contractor Report 172178, Institute for Computer Applications in Science and Engineering, NASA Langley Research Center, Hampton, Virginia 23665, 1983. 
ORNL/TM-12192

\section{INTERNAL DISTRIBUTION}

1. B.R. Appleton

2-3. T.S. Darland

4-8. E.F. D'Azevedo

9. J.M. Donato

10. J.J. Dongarra

11. G.A. Geist

12. M.R. Leuze

13. E.G. $\mathrm{Ng}$

14. C.E. Oliver

15. B.W. Peyton

16-20. S.A. Raby
21-25. C.H. Pomine

26. T.H. Rowan

27-31. R.F. Sincovec

32-36. R.C. Ward

37. P.H. Worley

38. Central Research Library

39. ORNL Patent Office

40. K-25 Appl 'Tech Library

41. Y-12 Technical Library

42. Lab Records Dept - RC

43-44. Laboratory Records Dept

\section{EXTERNAL DISTRIBUTION}

45. Cleve Ashcraft, Boeing Computer Services, P.O. Box 24346, M/S 7L-21, Seattle, WA 98124-0346

46. Donald M. Austin, 6196 EECS Bldg., University of Minnesota, 200 Union St., S.E., Minneapolis, MN 55455

47. Robert G. Babb, Oregon Graduate Institute, CSE Department, 19600 N.W. von Neumann Drive, Beaverton, OR 97006-1999

48. Lawrence J. Baker, Exxon Production Research Company, P.O. Box 2189, Houston, TX 77252-2189

49. Jesse L. Barlow, Department of Computer Science, Pennsylvania State University, University Park, PA 16802

50. Edward H. Barsis, Computer Science and Mathematics, P.O. Box 5800, Sandia National Laboratories, Albuquerque, NM 87185

51. Chris Bischof, Mathematics and Computer Science Division, Argonne National Laboratory, 9700 South Cass Avenue, Argonne, IL 60439

52. Ake Bjorck, Department of Mathematics, Linkoping University, S-581 83 Linkoping, Sweden

53. Jean R. S. Blair, Department of Computer Science, Ayres Hall, University of Tennessee, Knoxville, TN 37996-1301

54. Roger W. Brockett, Wang Professor of Electrical Engineering and Computer Science, Division of Applied Sciences, Harvard University, Cambridge, MA 02138

55. James C. Browne, Department of Computer Science, University of Texas, Austin, TX 78712

56. Bill L. Buzbee, Scientific Computing Division, National Center for Atmospheric Research, P.O. Box 3000, Boulder, CO 80307 
57. Donald A. Calahan, Department of Electrical and Computer Engineering, University of Michigan, Ann Arbor, MI 48109

58. John Cavallini, Deputy Director, Scientific Computing Staff, Applied Mathematical Sciences, Office of Energy Research, U.S. Department of Energy, Washington. DC 20585

59. Ian Cavers, Department of Computer Science, University of British Columbia, Vancouver, British Columbia V6T 1W5, Canada

60. Tony Chan, Department of Mathematics, University of California, Los Angeles, 405 Hilgard Avenue, Los Angeles, CA 90024

61. Jagdish Chandra, Army Research Office, P.O. Box 12211, Research Triangle Park, NC 27709

62. Eleanor Chu, Department of Mathematics and Statistics, University of Guelph, Guelph, Ontario, Canada N1G 2W1

63. Melvyn Ciment, National Science Foundation, 1800 G Street N.W., Washington, DC 20550

64. Tom Cc'eman, Department of Computer Science, Cornell University, Ithaca, NY 14853

65. Paul Concus, Mathematics and Computing, Lawrence Berkeley Laboratory, Berkeley, CA 94720

66. Andy Conn, IBM T. J. Watson Research Center, P.O. Box 218, Yorktown Heights, NY 10598

67. John M. Conroy, Supercomputer Research Center, 17100 Science Drive, Bowie, MD 20715-4300

68. Jane K. Cullum, IBM T. J. Watson Research Center, P.O. Box 218, Yorktown Heights, NY 10598

69. George Cybenko, Center for Supercomputing Research and Development, University of Illinois, $104 \mathrm{~S}$. Wright Street, Urbana, IL 61801-2932

70. George J. Davis, Department of Mathematics, Georgia State University, Atlanta, GA 30303

71. Tim A. Davis, Computer and Information Sciences Department, 301 CSE, University of Florida, Gainesville, FL 32611-2024

72. John J. Dorning, Department of Nuclear Engineering Physics, Thornton Hall, McCormick Road, University of Virginia, Charlottesville, VA 22901

73. Iain Duff, Atlas Centre, Rutherford Appleton Laboratory, Didcot, Oxon OX11 $0 Q X$, England

74. Patricia Eberlein, Department of Computer Science, SUNY at Buffalo, Buffalo, NY 14260

75. Stanley Eisenstat, Department of Computer Science, Yale University, P.O. Box 2158 Yale Station, New Haven, CT 06520

76. Lars Elden, Department of Mathematics, Linkoping University, 58183 Linkoping, Sweden 
77. Howard C. Elman, Computer Science Department, University of Maryland, College Park, MD 20742

78. Albert M. Erisman, Boeing Computer Services, Engineering Technology Applications, ETA Division, P.O. Box 24346, MS-7L-20 Seattle, WA 98124-0346

79. Geoffrey C. Fox, Northeast Parallel Architectures Center, 111 College Place, Syracuse University, Syracuse, NY 13244-4100

80. Paul O. Frederickson, NASA Ames Research Center, RIACS, M/S T045-1, Moffett Field, CA 94035

81. Fred N. Fritsch, L-316, Computing and Mathematics Research Division, Lawrence Livermore National Laboratory, P.O. Box 808, Livermore, CA 94550

82. Robert E. Funderlic, Department of Computer Science, North Carolina State University, Raleigh, NC 27650

83. K. Gallivan, Computer Science Department, University of Illinois, Urbana, IL 61801

84. Dennis B. Gannon, Computer Science Department, Indiana University, Bloomington, IN 47405

85. Feng Gao, Department of Computer Science, University of British Columbia, Vancouver, British Columbia V6T 1W5, Canada

86. David M. Gay, Bell Laboratories, 600 Mountain Avenue, Murray Hill, NJ 07974

87. C. William Gear, NEC Research Institute, 4 Independence Way, Princeton, NJ 08540

88. W. Morven Gentleman, Division of Electrical Engineering, National Research Council, Building M-50, Room 344, Montreal Road, Ottawa, Ontario, Canada K1A 0R8

89. J. Alan George, Vice President, Academic and Provost, Needles Hall, University of Waterloo, Waterloo, Ontario, Canada N2L 3G1

90. John R. Gilbert, Xerox Palo Alto Research Center, 3333 Coyote Hill Road, Palo Alto, CA 94304

91. Gene H. Golub, Department of Computer Science, Stanford University, Stanford, CA 94305

92. Joseph F. Grcar, Division 8245, Sandia Nationa! Laboratories, Livermore, CA 94551-0969

93. John Gustafson, Ames Laboratory, Iowa State University, Ames, IA 50011

94. Per Christian Hansen, UCI*C Lyngby, Building 305, Technical University of Denmark, DK-2800 Lyngby, Denmark

95. Richard Hanson, IMSL Inc., 2500 Park West Tower One, 2500 City West Blvd., Houston, TX 77042-3020

96. Michael T. Heath, National Center for Supercomputing Applications, 4157 Beckman Institute, University of Illinois, 405 North Mathews Avenue, Urbana, IL $61801-2300$

97. Don E. Heller, Physics and Computer Science Department, Shell Development Co., P.O. Box 481, Houston, TX 77001 
98. Nicholas J. Higham, Department of Mathematics, University of Manchester, Grt Manchester, M13 9PL, England

99. Charles J. Holland, Air Force Office of Scientific Research, Building 410, Bolling Air Force Base, Washington, DC 20332

100. Robert E. Huddleston, Computation Department, Lawrence Livermore National Laboratory, P.O. Box 808, Livermore, CA 94550

101. Ilse Ipsen, Department of Computer Science, Yale University, P.O. Box 2158 Yale Station, New Hayen, CT 06520

102. Barry Joe, Department of Computer Science, University of Alberta, Edmonton, Alberta T6G 2H1, Canada

103. Lennart Johnsson, Thinking Machines Inc., 245 First Street, Cambridge, MA 02142-1214

104. Harry Jordan, Department of Electrical and Computer Engineering, University of Colorado, Boulder, CO 80309

105. Bo Kagstrom, Institute of Information Processing, University of Umea, 5-901 87 Umea, Sweden

106. Malvyn H. Kalos, Cornell Theory Center, Engineering and Theory Center Bldg., Cornell University, Ithaca, NY 14853-3901

107. Hans Kaper, Mathematics and Computer Science Division, Argonne National Laboratory, 9700 South Cass Avenue, Bldg. 221, Argonne, IL 60439

108. Linda Kaufman, Bell Laboratories, 600 Mountain Avenue, Murray Hill, NJ 07974

109. Robert J. Kee, Division 8245, Sandia National Laboratories, Livermore, CA 94551 0969

110. Kenneth Kennedy, Department of Computer Science, Rice University, P.O. Box 1892, Houston, TX 77001

111. Thomas Kitchens, Department of Energy, Scientific Computing Staff, Office of Energy Research, ER-7, Office G-236 Germantown, Washington, DC 20585

112. Richard Lau, Office of Naval Research, Code 111MA, 800 Quincy Street, Boston Tower 1, Arlington, VA 22217-5000

113. Alan J. Laub, Department of Electrical and Computer Engineering, University of California, Santa Barbara, CA 93106

114. Robert L. Launer, Army Research Office, P.O. Box 12211, Research Triangle Park, NC 27709

115. Charles Lawson, MS 301-490, Jet Propulsion Laboratory, 4800 Oak Grove Drive, Pasadena, CA 91109

116. Peter D. Lax, Courant Institute of Mathematical Sciences, New York University, 251 Mercer Street, New York, NY 10012

117. James E. Leiss, Rt. 2, Box 142C, Broadway, VA 22815

118. John G. Lewis, Boeing Computer Services, P.O. Box 24346, M/S iL-21, Seattle, WA 98124-0346 
119. Jing Li, IMSL Inc., 2500 Park West Tower One, 2500 City West Blvd., Houston, TX 77042-3020

120. Heather M. Liddell, Center for Parallel Computing, Department of Computer Science and Statistics, Queen Mary College, University of London, Mile End Road, London E1 4NS, England

121. Arno Liegmann, c/o ETH Rechenzentrum, Clausiusstr. 55, CH-8092 Zurich, Switzerland

122. Joseph Liu, Department of Computer Science, York University, 4700 Keele Street, North York, Ontario, Canada M3J 1P3

123. Robert F. Lucas, Supercomputer Research Center, 17100 Science Drive, Bowie, MD 20715-4300

124. Franklin Luk, Department of Computer Science, Amos Eaton Building - \#131, Rensselaer Polytechnic Institute, Troy, NY 12180-3590

125. Thomas A. Manteuffel, Department of Mathematics, University of Colorado Denver, Campus Box 170, P.O. Box 173364, Denver, CO 80217-3364

126. Consuelo Maulino, Universidad Central de Venezuela, Escuela de Computacion, Facultad de Ciencias, Apartado 47002, Caracas 1041-A, Venezuela

127. James McGraw, Lawrence Livermore National Laboratory, L-306, P.O. Box 808, Livermore, CA 94550

128. Paul C. Messina, Mail Code 158-79, California Institute of Technology, $1201 \mathrm{E}$. California Blvd., Pasadena, CA 91125

129. Cleve Moler, The Mathworks, 325 Linfield Place, Menlo Park, CA 94025

130. Neville Moray, Department of Mechanical and Industrial Engineering, University of Illinois, 1206 West Green Street, Urbana, IL 61801

131. Dianne P. O'Leary, Computer Science Department, University of Maryland, College Park, MD 20742

132. James M. Ortega, Department of Applied Mathematics, Thornton Hall, University of Virginia, Charlottesville, VA 22901

133. Charles F. Osgood, National Security Agency, Ft. George G. Meade, MD 20755

134. Chris Paige, McGill University, School of Computer Science, McConnell Engineering Building, 3480 University Street, Montreal, Quebec, Canada H3A 2A7

135. Roy P. Pargas, Department of Computer Science, Clemson University, Clemson, SC 29634-1906

136. Beresford N. Parlett, Department of Mathematics, University of California, Berkeley, CA 94720

137. Merrell Patrick, Department of Computer Science, Duke University, Durham, NC 27706

138. Robert J. Plemmons, Departments of Mathematics and Computer Science, Box 7311, Wake Forest University, Winston-Salem, NC 27109

139. Jesse Poore, Department of Computer Science, Ayres Hall, University of Tennessee, Knoxville, TN 37996-1301 
140. Alex Pothen, Department of Computer Science, Pennsylvania State University, University Park, PA 16802

141. Yuanchang Qi, IBM European Petroleum Application Center, P.O. Box 585, N4040 Hafrsfjord, Norway

142. Giuseppe Radicati, IBM European Center for Scientific and Engineering Computing, via del Giorgione 159, 1-00147 Roma, Italy

143. John K. Reid, Numerical Analysis Group, Central Computing Department, Atlas Centre, Rutherford Appleton Laboratory, Didcot, Oxon OX11 0QX, England

144. Werner C. Rheinboldt, Department of Mathematics and Statistics, University of Pittsburgh, Pittsburgh, PA 15260

145. John R. Rice, Computer Science Department, Purdue University, West Lafayette, IN 47907

146. Garry Rodrigue, Numerical Mathematics Group, Lawrence Livermore Laboratory, Livermore, CA 94550

147. Donald J. Rose, Department of Computer Science, Duke University, Durham, NC. 27706

148. Edward Rothberg, Department of Computer Science, Stanford University, Stanford, CA 94305

149. Axel Ruhe, Dept. of Computer Science, Chalmers University of Technology, S41296 Goteborg, Sweden

150. Joel Saltz, ICASE, MS 132C, NASA Langley Research Center, Hampton, VA 23665

151. Ahmed H. Sameh, Center for Supercomputing R\&D, 1384 W. Springfield Avenue, University of Illinois, Urbana, IL 61801

152. Michael Saunders, Systems Optimization Laboratory, Operations Research Department, Stanford University, Stanford, CA 94305

153. Robert Schreiber, RIACS, Mail Stop 230-5, NASA Ames Research Center, Moffet Field, CA 94035

154. Martin H. Schultz, Department of Computer Science, Yale University, P.O. Box 2158 Yale Station, New Haven, CT 06520

155. David S. Scott, Intel Scientific Computers, 15201 N.W. Greenbrier Parkway, Beaverton, OR 97006

156. Lawrence F. Shampine, Mathematics Department, Southern Methodist University, Dallas, TX 75275

157. Andy Sherman, Department of Computer Science, Yale University, P.O. Box 2158 Yale Station, New Haven, CT 06520

158. Kermit Sigmon, Department of Mathematics, University of Florida, Gainesville, FL 32611

159. Horst Simon, Mail Stop T045-1, NASA Ames Research Center, Moffett Field, CA 94035

160. Anthony Skjellum, Lawrence Livermore National Laboratory, 7000 East Ave., L316, P.O. Box 808 Livermore, CA 94551 
161. Danny C. Sorensen, Department of Mathematical Sciences, Rice University, P.O. Box 1892, Houston, TX 77251

162. G. W. Stewart, Computer Science Department, University of Maryland, College Park, MD 20742

163. Paul N. Swartztrauber, National Center for Atmospheric Research, P.O. Box 3000, Boulder, CO 80307

164. Philippe Toint, Dept. of Mathematics, University of Namur, FUNOP, 61 rue de Bruxelles, B-Namur, Belgium

165. Bernard Tourancheau, LIP, ENS-Lyon, 69364 Lyon cedex 07, France

166. Hank Van der Vorst, Dept. of Techn. Mathematics and Computer Science, Delft University of Technology, P.O. Box 356, NL-2600 AJ Delft, The Netherlands

167. Charles Van Loan, Department of Computer Science, Cornell University, Ithaca, NY 14853

168. Jim M. Varah, Centre for Integrated Computer Systems Research, University of British Columbia, Office 2053-2324 Main Mall, Vancouver, British Columbia V6T 1W5, Canada

169. Udaya B. Vemulapati, Dept. of Computer Science, University of Central Florida, Orlando, FL 32816-0362

170. Robert G. Voigt, ICASE, MS 132-C, NASA Langley Research Center, Hampton, VA 23665

171. Phuong Vu, Cray Research, Inc., 19607 Franz Rd., Houston, TX 77084

172. Daniel D. Warner, Department of Mathematical Sciences, O-104 Martin Hall, Clemson University, Clemson, SC 29631

173. Mary F. Wheeler, Rice University, Department of Mathematical Sciences, P.O. Box 1892, Houston, TX 77251

174. Andrew B. White, Computing Division, Los Alamos National Laboratory, P.O. Box 1663, MS-265, Los Alamos, NM 87545

175. Margaret Wright, Bell Laboratories, 600 Mountain Avenue, Murray Hill, NJ 07974

176. David Young, University of Texas, Center for Numerical Analysis, RLM 13.150, Austin, TX 78731

177. Earl Zmijewski, Department of Computer Science, University of California, Santa Barbara, CA 93106

178. Office of Assistant Manager for Energy Research and Development, U.S. Department of Energy, Oak Ridge Operations Office, P.O. Box 2001 Oak Ridge, TN $37831-8600$

179-188. Office of Scientific \& Technical Information, P.O. Box 62, Oak Ridge, TN 37831 

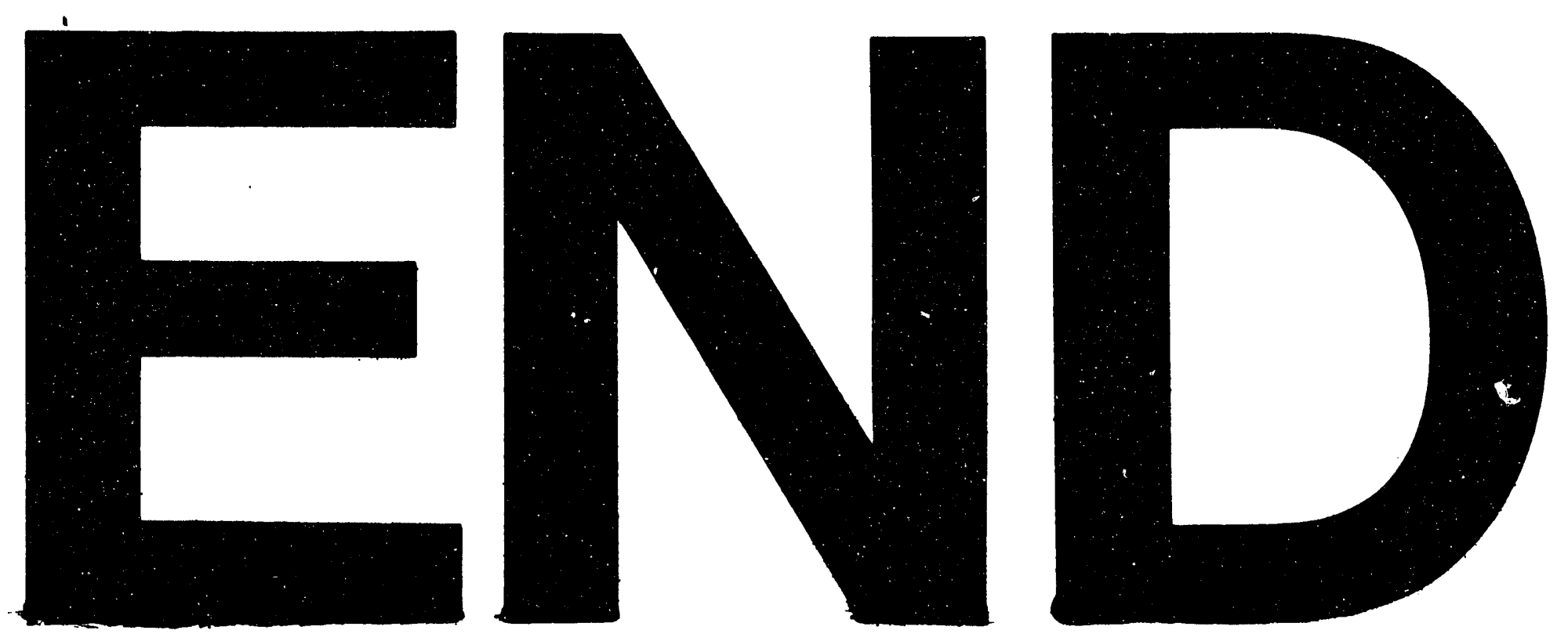
$\because$

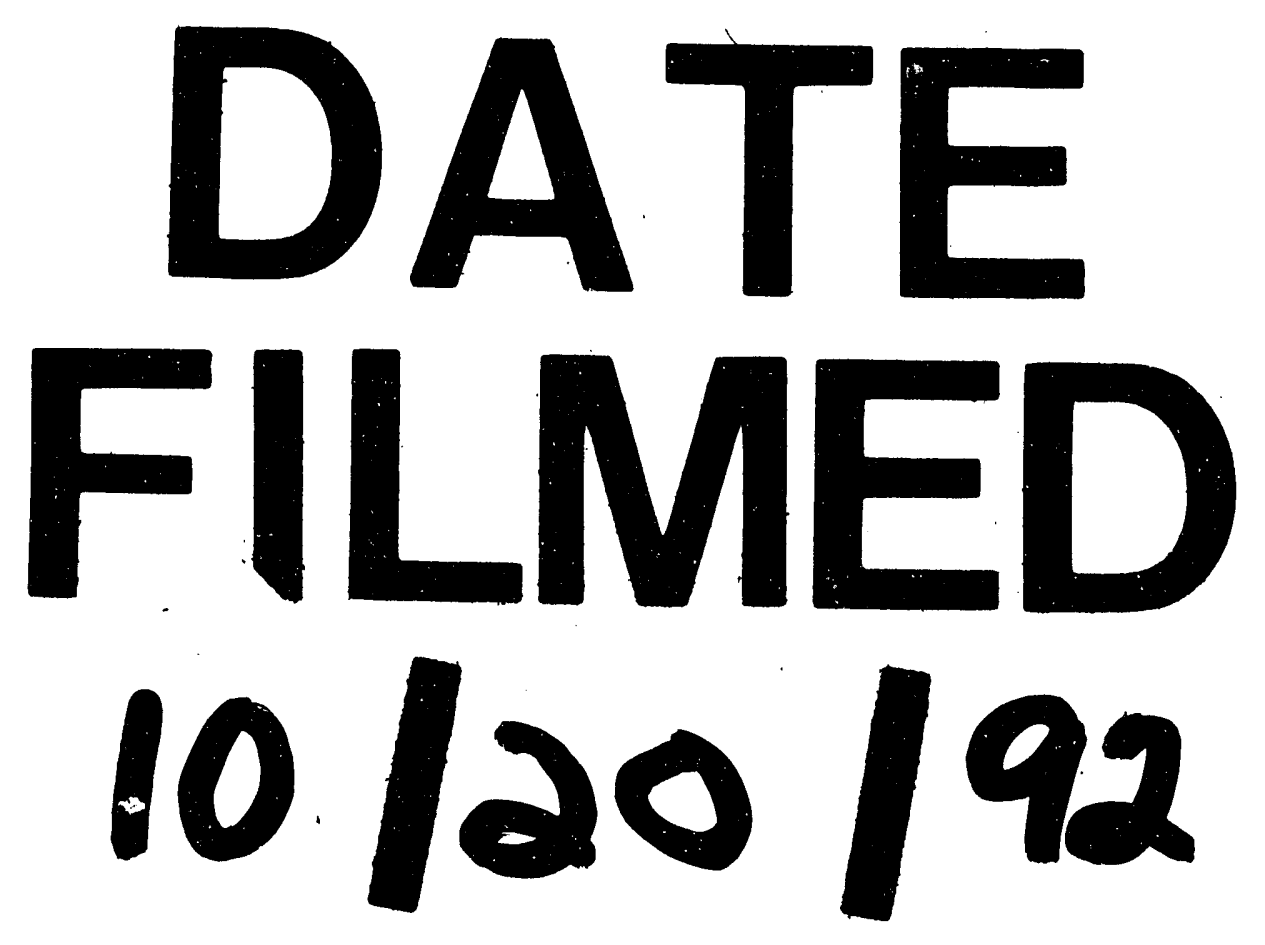

